\title{
Time-Frequency Analysis of 24-Hour Heart Rate Fluctuation for the Characterization of Very Low-Frequency Component
}

\author{
Hsiao-Lung Chan ${ }^{\dagger}$, Jiunn-Lee Lin ${ }^{\ddagger}$, Chao-Cheng Du ${ }^{\ddagger}$, and Chien-Ping Wu ${ }^{\dagger}$ \\ $\dagger$ Department of Electrical Engineering, $\ddagger_{\text {Department of Internal Medicine }}$ \\ National Taiwan University, Taipei, Taiwan, Republic of China
}

\begin{abstract}
A smooth pseudo Wigner-Ville distribution was employed to investigate both time and frequency characteristics in very low-frequency component (less than $0.04 \mathrm{~Hz}$ ) of 24-hour heart rate fluctuation. In some of heart failure, we could see remarkable and very lowfrequency oscillations on the Wigner-Ville distribution, which are related to Cheyne-Stokes respiration. However, the Wigner-Ville distribution of normal subjects had not such oscillations, but more random spectral characteristics instead. Furthermore, we calculated $1 / f^{\alpha}$ regression slopes of average spectrum to be a crude estimate of fractal dimensions. The slopes are more negative in heart failure than normal $(-1.866 \pm 0.888$ vs. $-0.957 \pm 0.180 p<$ 0.005 ), which associates a loss of dimensionality of heart rate fluctuation in heart failure.
\end{abstract}

\section{INTRODUCTION}

Spectral analysis of heart rate variability provides a way to understand the different control mechanisms of the autonomic nervous system. In previous researches[1], the high frequency fluctuations of heart rate (approximately 0.15-0.40 $\mathrm{Hz}$ ) were regarded as vagal control, and were correlated with respiration. And, the low frequency fluctuations (approximately 0.04-0.15 $\mathrm{Hz}$ ) were due to both sympathetic and vagal control. However, for the implicated mechanism of very low-frequency fluctuations (less than $0.04 \mathrm{~Hz}$ ), there were some hypothesis and observations, such as renin-angiotension regulation [1], temperature regulation [2], a chaotic behavior[3], and a longwavelength oscillation in heart failure[4]. In clinical study, power in very low-frequency band differentiated the heart failure group from the normal group[5]. Moreover, it had strong association with mortality after myocardial infarction[6]. Thus, we regarded it might imply some physiological or pathological information in very low-frequency heart rate.

One problem exists in the analysis of very-low frequency heart rate : what is the proper size of time window for spectral analysis? A particular window should be appropriate for a particular signal to give better frequency resolution. Longer window could provide better frequency resolution, but would smear the time-varying characteristic of signals (ie, poor time resolution). To overcome this problem, we employed Wigner-Ville distribution to reveal possible phenomenons in very low-frequency band of 24-hour heart rate.

\section{METHODS}

\section{A. Subjects and preliminary processing}

Thirteen heart failure patients and fourteen normal subjects were included for this study. All subjects underwent 24-hour Electrocardiogram recording. ECG signals were digitized into $\mathrm{PC}$ for QRS detection and verification. The instantaneous heart rate were equal-sampling $(2 \mathrm{~Hz})$ using cubic spline interpolation. In order to reduce the heavy computation in Wigner-Ville distribution, the sampling rate of heart rate was further reduced to 0.2 $\mathrm{Hz}$. Before subsampling, original heart rate were filtered with a FIR low-pass filter with $0.08 \mathrm{~Hz}$ cut-off frequency to avoid aliasing.

\section{B. Smooth pseudo Wigner-Ville distribution (SPWVD)}

The SPWVD of a heart rate signal $s(n)$ is expressed as follows :

$$
\begin{gathered}
S P W V D(n, k)=\sum_{i=-N+1}^{N-1}|h(i)|^{2}\left[\sum_{m=-M+1}^{M-1} g(m)\right. \\
\left.z(n+m+i) z^{*}(n+m-i)\right] \exp \left(-j \frac{2 \pi}{N} i k\right)
\end{gathered}
$$

where $h(i)$ is a Gausian window, $g(m)$ is a rectangular window, and $z(n)$ is the analytic signal associated with real heart rate signal $s(n)$. It is calculated in the time dormain as follows :

$$
z(n)=s(n)+j H[s(n)]
$$

where $H[$.$] is Hilbert transform.$

Using windows of $h(i)$ and $g(m)$, it allows independent smoothing in both directions, frequency and time by selecting different window size, $2 N-1$ and $2 M-1$ respectively $[7,8]$. Also, it can reduce cross terms which may encounter in WVD. The use of the analytic signal could avoid low-frequency artifacts and aliasing components produced in the real Wigner distribution [9].

\section{C. $1 / f^{\alpha}$ regression slope}

In order to study the fractal dimensions of heart rate fluctuation, the average spectrum, time integration of Wigner-Ville distribution, were characterized as an inverse power-law $\left(1 / f^{\alpha}\right.$-like) spectrum within the following frequency band : the highest frequency was set to $0.04 \mathrm{~Hz}$, 
and the lowest frequency was the peak frequency associated maximum power below $0.04 \mathrm{~Hz}$. With steeper slope $\alpha$, the fractal dimension of heart rate fluctuation would be smaller, and vice versa.

\section{RESULTS}

Figure 1 shows the Wigner-Ville distribution of 24hour heart rate fluctuation in a heart failure patient. One can easily see remarkable and very low-frequency oscillations during night, and more random spectrum in the other time. These oscillations are regarded as CheyneStokes respiration (CSR)[3]. It implies that high degree freedom of heart rate fluctuation have become locked into a single wavelength during CSR. Meanwhile, the oscillation frequency, amplitude, duration, and occurrence time could be identified. However, the Wigner-Ville distribution of normal subjects have not such oscillations, but more random spectral characteristics instead.

Comparing regression slopes of average spectrum, we found it has statistical differences $(-1.866 \pm 0.888 \mathrm{vs}$. $0.957 \pm 0.180 p<0.005)$. This result demonstrats that heart failure subjects yield more negative slopes than normal subjects, that is, it is associated with a loss of dimensionality of heart rate fluctuation in heart failure.

\section{DISCUSSION}

Very Low-frequency power of heart rate accounts most portion of total power in the spectrum of 24-hour heart rate. Unlike other higher frequency power, it is less directly related to the action of vagal or sympathetic system. By the tranform of 24 heart rate to Wigner-Ville distribution, it not only provides more high-resolution spectral characteristics, but also reveals both frequencyvarying and time-varying characteristics of signal. Therefore, it will be helpful for observing the very low-frequency dynamics of the regualtion of the cardiovascular system.

Although there would exist cross terms in the WignerVille distribution, these could be improved by appropriate selection of smoothing window $(g(m) \& h(i))$ [8]. In addition, the computation work of this SPWVD is less heavy, compared with other interference-reduced WVD [9]. So it is suitable for signal with a large amount of data, such as 24 heart rate fluctuation signal.

\section{ACKNOWLEDGEMENT}

We are grateful for the support of grants NSC 820404-E002-397 from the National Science Council, Taiwan, Republic of China.

\section{REFERENCES}

[1] S.Akselrod, et al, " Power spectrum analysis of heart rate fluctuation : A quantitative probe of beat-to-beat cardiovascular control." Science, 213, pp.220-223, 1981.

[2] B.Sayers, "Analysis of heart rate variabilty.", Ergonomics, 16, pp.17-32, 1973.

[3] M.Kobayashi, et al, "1/f fluctuatuion of heartbeat period.", IEEE Trans. Biomed. Eng., Vol.29, No.6, pp.456$457,1982$.

[4] A.L.Goldberger, et al, "Nonlinear dynamics in heart failure : Implications of long-wavelength cardiopulmonary oscillations." , Am. Heart J., Vol.107, No.3, pp.612-615, 1984.
[5] J.P.Saul, et al, "Assessment of autonomic regulation in chronic congestive heart failure by heart rate spectral analysis.", Am. J. Cardiol., 61, pp.1292-1299, 1988.

[6] J.T.Bigger, et al, "Frequency domain measures of heart period variability and mortality after myocardial infarction.", Circulation, Vol.85, No.1, January, pp.164-171, 1992.

[7] W.Martin, et al, "Wigner-Ville spectral analysis of nonstationary process.", IEEE Trans. ASSP Vol.33, No.4, Dec., pp.1461-1470, 1985.

[8] P.Novak, et al, " Time/frequency mapping of the heart rate, blood pressure and respiratory signals", Med. Biol. Eng. Comput., 31, pp.103-110, 1993.

[9] B.Boashash, "Time-frequency siganl analysis", Advances in Spectrum Analysis and Array Processing, Vol.I, Chap.9, Prentice-Hall Inc., 1991.

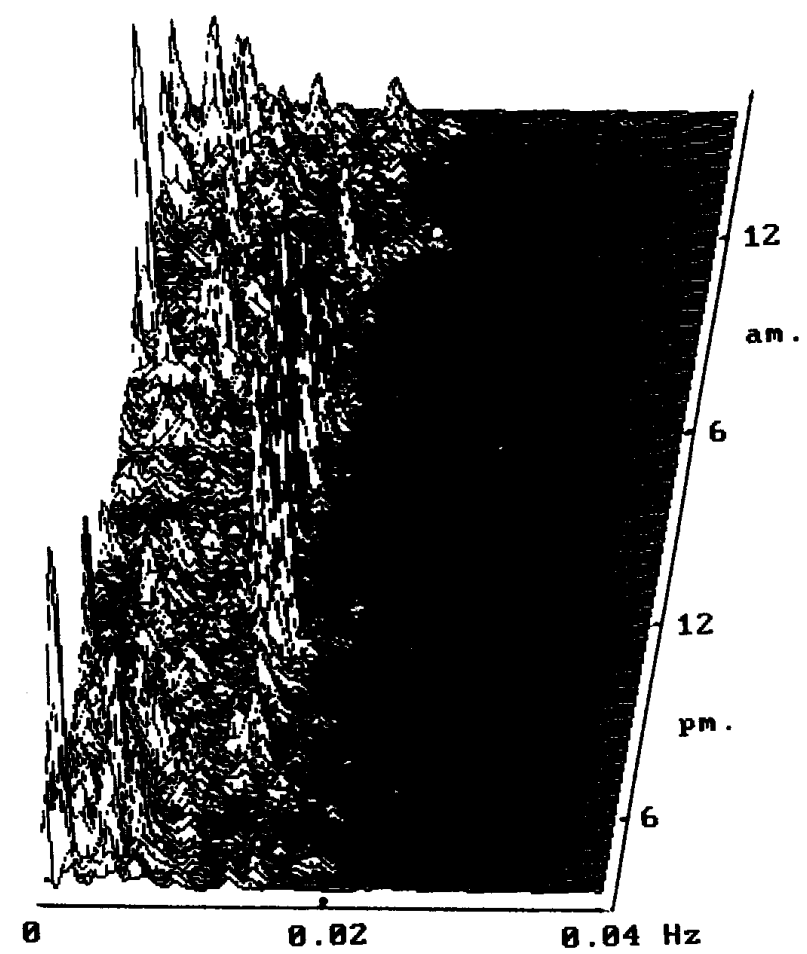

Figure 1: SPWVD of one heart failure patient. There are about $0.015 \mathrm{~Hz}$ low frequency oscillations during night, and are regarded as Cheyne-Stokes respiration. 